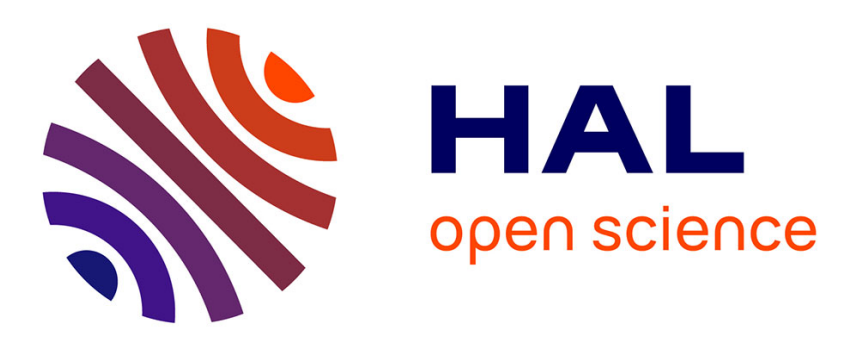

\title{
Compressed Raman classification method with upper-bounded error probability
} Philippe Réfrégier, Emmanuel Chevallier, Frédéric Galland

\section{To cite this version:}

Philippe Réfrégier, Emmanuel Chevallier, Frédéric Galland. Compressed Raman classification method with upper-bounded error probability. Optics Letters, 2019, 44 (23), pp.5836. 10.1364/OL.44.005836 . hal-02394203

\section{HAL Id: hal-02394203 \\ https://hal.science/hal-02394203}

Submitted on 6 Jan 2021

HAL is a multi-disciplinary open access archive for the deposit and dissemination of scientific research documents, whether they are published or not. The documents may come from teaching and research institutions in France or abroad, or from public or private research centers.
L'archive ouverte pluridisciplinaire HAL, est destinée au dépôt et à la diffusion de documents scientifiques de niveau recherche, publiés ou non, émanant des établissements d'enseignement et de recherche français ou étrangers, des laboratoires publics ou privés. 


\title{
A compressed Raman classification method with upper bounded error probability
}

\author{
Philippe Réfrégier ${ }^{1}$, Emmanuel Chevallier ${ }^{1}$, And Frédéric Galland ${ }^{1^{*}}$ \\ ${ }^{1}$ Aix Marseille Univ, CNRS, Centrale Marseille, Institut Fresnel, Marseille, France \\ ${ }^{*}$ Corresponding author: frederic.galland@fresnel.fr
}

Preprint version

\begin{abstract}
Classification of different species with Raman measurements is analyzed when a total of exactly $N$ photons are detected with binary filtered Raman spectra instead of fixing the measuring time. The optimal classification method for this problem leads to classification error probabilities upper bounded by the Bhattacharyya bound and that are invariant to the multiplication of the spectrum intensities by an unknown factor. Furthermore, it is shown that this approach can be implemented with a number of binary filters smaller than the number of species to discriminate.
\end{abstract}

OCIS codes: $\quad 070.4560$ Data processing by optical means 110.4280 Noise in imaging systems 110.3055 Information theoretical analysis 070.5010 Pattern recognition.

http://dx.doi.org/10.1364/OL.44.005836

Raman spectrum can be used to classify different species. In order to limit measuring times and to implement photon limited sensors, it has been proposed to only measure few characteristics obtained by optically filtering the Raman spectrum [111]. It has also been shown [1-11] that this approach can lead to measurements limited by photon noise with small measuring times. These techniques, also known as compressive Raman detection [3-6, 8-11], or compressed methods [12] can be implemented with binary filters (BF). Indeed, it leads to simpler technological constraints than using continuous valued filters with, nevertheless, very good performance [12]. In the previous compressive methods with BF [1-13], the light is transmitted or blocked out for each frequency channel and the classification between the different species is performed with the number of photons detected during a fixed measuring time. However, there exists situations for which it is necessary to control the classification error probability without knowing a priori the mean number of the emitted photons but only of the normalized Raman spectra. In this Letter, this problem is addressed with the alternative technique that consists of measuring the number of photons detected after each BF until the total number of detected photons has reached an a priori fixed value $N$. More precisely, let us assume that a set of $P$ mathematically orthogonal BF (OBF) are implemented (orthogonality means physically that there is no overlap between the filters). Let $n_{m}$, denote the number of photons detected on detector $D_{m}$ after the light has been filtered by the OBF number $m$. The measurements are thus performed until that $n_{1}+n_{2}+\ldots+n_{P}=N$.

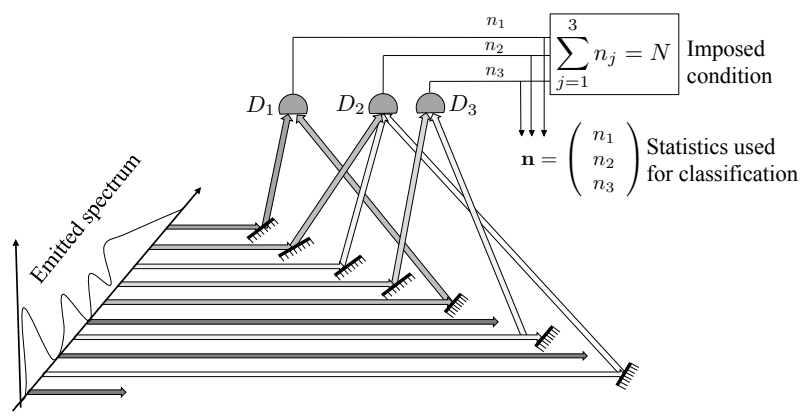

Fig. 1. Schematic representation of the considered detecting system when $P=3$. Orthogonal Binary Filters are assumed implemented with an optical perfect shuffle.

Let $\tau$ denote the measuring time and let $u$ denote the analyzed species. In the following, full Raman technique corresponds to the situation for which the whole spectrum is measured with $K$ bins. In that case, the average number of photons that could be detected for frequencies $v \in\left[v_{k}-\delta_{v} / 2, v_{k}+\delta_{v} / 2\right]$, with $k=1,2, \ldots, K$, will be denoted $S^{(u)}\left(v_{k}\right) \tau$. It is assumed that $v_{k+1}-v_{k}>\delta_{v}$, i.e. that the frequency bins are non overlapping. A set of $P$ OBF $[9,12,13], \mathbf{F}_{m}$ (with $m=1, . ., P$ ) is defined so that $F_{m}\left(v_{k}\right)=0$ or 1 and $\sum_{k=1}^{K} F_{m}\left(v_{k}\right) F_{n}\left(v_{k}\right)=0$ (see schematic representation in Fig. 1). Thus at most one BF does not block out each frequency bin. The mathematical orthogonality of OBF thus allows one to potentially implement them in parallel and opens also new perspectives for experimental strategies. In that case, the mean number of measured photons on $D_{m}$ after OBF $\mathbf{F}_{m}$ is thus $\mu_{m}^{(u)}=\phi_{m}^{(u)} \tau$ where:

$$
\phi_{m}^{(u)}=\sum_{k=1}^{K} F_{m}\left(v_{k}\right) S^{(u)}\left(v_{k}\right)
$$

Thus, knowing that a photon has been detected, the probability that it has been detected on $D_{m}$ is:

$$
p_{m}^{(u)}=\frac{\mu_{m}^{(u)}}{\sum_{m=1}^{P} \mu_{m}^{(u)}}=\frac{\sum_{k=1}^{K} F_{m}\left(v_{k}\right) S^{(u)}\left(v_{k}\right)}{\sum_{m=1}^{P} \sum_{k=1}^{K} F_{m}\left(v_{k}\right) S^{(u)}\left(v_{k}\right)}
$$


This quantity is independent of $\tau$ and is invariant if the spectrum intensity is multiplied by an unknown factor (i.e. if $\left.S^{(u)}\left(v_{k}\right) \rightarrow a S^{(u)}\left(v_{k}\right)\right)$. Let $\boldsymbol{n}=\left(n_{1}, n_{2}, \ldots, n_{P}\right)$, then, the probability to observe $n$ for species $u$ knowing that a total of $N$ photons have been detected (i.e. that $\sum_{m=1}^{P} n_{m}=N$ ) is:

$$
P(\boldsymbol{n} \mid u, N)=N ! \prod_{m=1}^{P} \frac{\left[p_{m}^{(u)}\right]^{n_{m}}}{n_{m} !}
$$

which corresponds to a multinomial distribution and where $x !=1 \times 2 \times 3 \ldots \times x$. All along this letter, it will be assumed that each class $u$ has the same probability to occur, i.e. $P(u)=1 / M$ where $M$ is the number of classes. Thus, the classification method that minimizes the classification error probability [14] is the value $\widehat{u}^{o p t}$ of $u$ that maximizes $\log [P(\boldsymbol{n} \mid u, N)]$, which can be written $\widehat{u}^{o p t}=\arg \max _{u} \log [P(\boldsymbol{n} \mid u, N)]$ and which leads to:

$$
\widehat{u}^{\text {opt }}=\arg \max _{u}\left[\sum_{m=1}^{P} n_{m} \log \left(p_{m}^{(u)}\right)\right]
$$

Let us first discuss the case of classification between two classes $u$ and $v$ (and thus, in that case $P(u)=P(v)=1 / 2$ ). When optimal classification methods are implemented, it can be shown that the classification error probability is necessarily smaller than bounds that are often easier to determine than the classification error probability itself [14]. In particular the classification error probability $\mathcal{P}_{u, v}(\mathcal{E})$ of the optimal method of Eq. (4) is upper bounded by the Bhattacharyya bound (BB) [1316]:

$$
\mathcal{P}_{u, v}(\mathcal{E}) \leq \frac{1}{2} e^{-\mathcal{B}_{u, v}}
$$

where the Bhattacharyya distance is:

$$
\mathcal{B}_{u, v}=-\log \left[\sum_{\boldsymbol{n} \in \Omega_{N}} \sqrt{P(\boldsymbol{n} \mid u, N) P(\boldsymbol{n} \mid v, N)}\right]
$$

where $\Omega_{N}$ is the set of $n$ values so that $n_{m} \geq 0$ and $\sum_{m=1}^{P} n_{m}=$ $N$. A simple calculus shows that:

$$
\mathcal{B}_{u, v}=-N \log \left[\sum_{m=1}^{P} \sqrt{p_{m}^{(u)} p_{m}^{(v)}}\right]
$$

With $\boldsymbol{q}_{u}^{T}=\left(\sqrt{p_{1}^{(u)}}, \sqrt{p_{2}^{(u)}}, \ldots \sqrt{p_{P}^{(u)}}\right)$, the BB can be written:

$$
\mathcal{P}_{u, v}(\mathcal{E}) \leq \frac{1}{2}\left(\boldsymbol{q}_{u} \cdot \boldsymbol{q}_{v}\right)^{N}
$$

where $\boldsymbol{q}_{u} \cdot \boldsymbol{q}_{v}$ is the scalar product between $\boldsymbol{q}_{u}$ and $\boldsymbol{q}_{v}$. Since the norm $\left\|\boldsymbol{q}_{u}\right\|$ of $\boldsymbol{q}_{u}$ satisfies $\left\|\boldsymbol{q}_{u}\right\|=1$, the scalar product $\boldsymbol{q}_{u} \cdot \boldsymbol{q}_{v}$ has a simple geometrical interpretation on the part of the sphere (which is a circle if $P=2$ and an hypersphere is $P \geq 4$ ) defined by $q_{m}^{(u)} \geq 0$ and $\sum_{m=1}^{P}\left|q_{m}^{(u)}\right|^{2}=1$.

Let us first illustrate the relevance of the BB when $P=M=$ 2 and when each species has the same probability to occur. In that case, $n_{1}$ has a binomial distribution with parameter $p_{u}$ for species $u$ and $n_{2}=N-n_{1}$. The classification error probabilities can thus be estimated considering realizations of random variables with binomial distributions with parameter $p_{u}$ for species $u$ or with parameter $p_{v}$ for species $v$. Simulation results are shown in Fig. 2 when $N=20$. The classification error probabilities have been estimated with $N_{e}=10^{3}$ independent realizations of random variables $n_{1}$ for each class $u$ and $v$. Such estimations have been performed for $N_{p}=50$ different values of $p_{u}$ and $p_{v}$ that are realizations of random numbers uniformly distributed in $[0,1]$. The results are shown in Fig.2 as a function of $\frac{1}{2} e^{-\mathcal{B}_{u, v}}$.

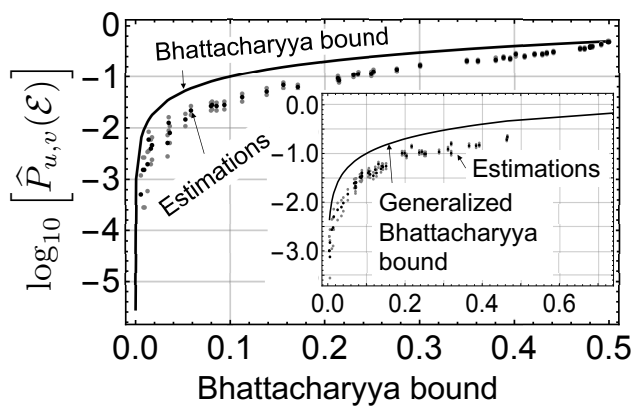

Fig. 2. Decimal logarithm of the estimated classification error probability $\widehat{\mathcal{P}}_{u, v}(\mathcal{E})$ with $N_{e}=10^{3}$ independent realizations of binomial random variables when $N=20$ photons are detected and for $N_{p}=50$ random laws $\left(p_{u}, p_{v}\right)$, where $p_{u}$ and $p_{v}$ are independent realizations of uniformly distributed random variables in $[0,1]$. It is assumed that $P=2$ (i.e. $2 \mathrm{OBF}$ are implemented) for 2 classes. $\widehat{\mathcal{P}}_{u, v}(\mathcal{E})$ are shown in Black. Gray points show these values plus or minus the standard deviation of estimation (estimated with $\sqrt{\widehat{\mathcal{P}}_{u, v}(\mathcal{E})\left[1-\widehat{\mathcal{P}}_{u, v}(\mathcal{E})\right]} / \sqrt{N_{e}}$ ). The continuous curve shows the Bhattacharyya bound. Insert: The same but it is now assumed that $N=50$ and that $P=4$ OBF are implemented for 4 classes and where the generalized Bhattacharyya bound is provided by Eq. (11). In that case, $p_{m}^{(u)}=\frac{y_{m}^{(u)}}{\sum_{m=1}^{p} y_{m}^{(u)}}$ where $y_{m}^{(u)}$ are independent realizations of random variables uniformly distributed in $[0,1]$.

Let $\mathcal{N}_{u}=\Phi_{u} \tau_{u}=\sum_{k=1}^{K} S^{(u)}\left(v_{k}\right) \tau_{u}$, where $\tau_{u}$ is the mean measuring time in order to get $N$ photons on the detectors for species $u$. Thus, $\mathcal{N}_{u}$ is the equivalent photon numbers of the light emitted by the sample of species $u$ before the OBF for this measuring time $\tau_{u}$. The total mean number of detected photons after the OBF is in that case $\phi_{u} \tau_{u}$ with $\phi_{u}=\sum_{m=1}^{2} \phi_{m}^{(u)}$. The ratio $\eta_{O B F}=\left(\phi_{u} \tau_{u}+\phi_{v} \tau_{v}\right) /\left(\Phi_{u} \tau_{u}+\Phi_{v} \tau_{v}\right)$ is thus equivalent to an optical efficiency of the BF. Synthesizing OBF by maximizing Eq. (7) can lead to low optical efficiency $\eta_{O B F}$. In such a case, the measurement can require the emission of a high number of photons by the sample before getting $N$ photons on the detector. It can thus be useful to impose that $\mathcal{N}_{u}+\mathcal{N}_{v}$, or equivalently $\Phi_{u} \tau_{u}+\Phi_{v} \tau_{v}$, is upper bounded. Moreover, when the spectrum models have been normalized so that $\Phi_{u}=\Phi_{v}=\Phi$, this is equivalent to impose a constraint on $T=\left(\tau_{u}+\tau_{v}\right) / 2$. Furthermore, $N=\phi_{u} \tau_{u}$ and then:

$$
\tau_{u}=\frac{N}{\sum_{m=1}^{2} \phi_{m}^{(u)}}=\frac{N}{\sum_{m=1}^{2} \sum_{k=1}^{K} F_{m}\left(v_{k}\right) S^{(u)}\left(v_{k}\right)}
$$

In the following, results are illustrated with spectra of the form:

$$
S^{(u)}\left(v_{k}\right)=A_{u, \alpha}\left(X_{u, k}\right)^{\alpha}
$$

where $X_{u, k}$ are real positive random numbers distributed with a unit mean exponential probability density function, where $\alpha>$ 0 , where $A_{u, \alpha}$ is defined so that $\sum_{k=1}^{K} S^{(u)}\left(v_{k}\right)=1$ (i.e. $A_{u, \alpha}^{-1}=$ $\left.\sum_{k=1}^{K}\left(X_{u, k}\right)^{\alpha}\right)$ and where $K=50$. The exponent $\alpha$ allows one 
to simulate spectra with different correlations characterized by $\boldsymbol{q}_{u} \cdot \boldsymbol{q}_{v}([13])$

The design of the filters is obtained here with a simple optimization technique. This technique consists, for each $v_{k}$, to chose a new value of $\left[F_{1}\left(v_{k}\right), F_{2}\left(v_{k}\right)\right]$ in $[(0,0),(0,1),(1,0)]$ and to accept it only if it increases $\mathcal{B}_{u, v}$ and satisfies $T=\left(\tau_{u}+\right.$ $\left.\tau_{v}\right) / 2 \leq T_{\lambda}$. Let $T_{F}$ corresponds to the obtained value of $T$ with the OBF that maximize $\mathcal{B}_{u, v}$ (Eq. (7)) without constraint on $T$. Then, in Fig. 3, the optimizations have been realized for seven maximal imposed values $T_{\lambda}$ of $T$ so that $T_{\lambda}=\lambda T_{F}$ with $\lambda \leq 1$. The process is repeated a fixed number of times (2000 in the shown numerical experiments). Since the obtained results are sensitive to the initialization, the result with the highest $\mathcal{B}_{u, v}$ was selected among 20 different initial couples of OBF. These initial OBF were chosen so that $\sum_{m=1}^{2} F_{m}\left(v_{k}\right)=1$ for any frequency bin $v_{k}$. Fig. 3 shows that the optimized Bhattacharyya distance strongly depends on the imposed $T_{\lambda}$ value and, of course, on $\alpha$.

For two classes, the ratio $\mathcal{B}_{u, v} / T$ is a criterion independent of $N$ as shown with Eq. (7) and Eq. (9). Let $\mathbf{F}^{(0)}$ denote the set of $P$ OBF obtained by maximizing $\mathcal{B}_{u, v} / T$ and let $\mathbf{F}^{\left(T_{1}\right)}$ denote the set of $P$ OBF that has been obtained by maximizing $\mathcal{B}_{u, v}$ with $N=1$ with the constraint that $T \leq T_{1}$. To each set of $P$ OBF corresponds a point in the [time, $\mathrm{BB}]$ diagram analogous to the one of Fig. 3. Points obtained with $\mathbf{F}^{(0)}$ when applied with different $N$ values are located on a straight line in this diagram since both $\mathcal{B}_{u, v}$ and $T$ are proportional to $N$. The point in the [time, BB] diagram for any set of filters $\mathbf{F}^{\left(T_{1}\right)}$ cannot be located on the left side of this straight line. Indeed, otherwise there will be a contraction with the property that $\mathbf{F}^{(0)}$ maximizes $\mathcal{B}_{u, v} / T$. Since the mean number of equivalent emitted photons before the OBF is $\mathcal{N}=\left(\mathcal{N}_{u}+\mathcal{N}_{v}\right) / 2=\Phi T$, if several sets of OBF leads to the same $\mathrm{BB}$, the one with the smallest $T$ value corresponds to the one that leads to a minimal mean number of equivalent emitted photons before the OBF. From that point of view, the OBF couple $\mathbf{F}^{(0)}$ leads to a good trade-off.

Results obtained with this approach are shown in Fig.3 for three values of $\alpha$. It can be observed that the optimization of $\mathcal{B}_{u, v} / T$ leads to a trade-off between $\mathcal{B}_{u, v}$ and $T$ that is not improved by maximizing $\mathcal{B}_{u, v}$ with imposed $T_{\lambda}$ values.

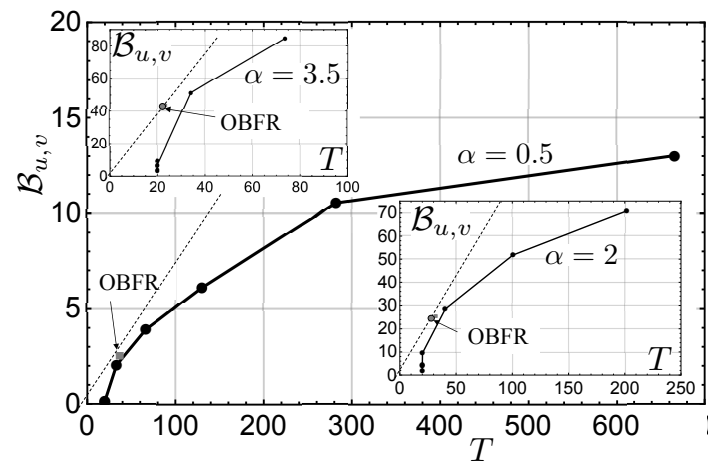

Fig. 3. Bhattacharyya distance $\mathcal{B}_{u, v}$ as a function of the mean measuring time $T$ for classification between two classes with couples of spectra with exponent $\alpha=0.5,2,3.5$ and with $P=2$ OBF. Gray points (OBFR) show the obtained results with optimization of $\mathcal{B}_{u, v} / T$. Continuous and dashed lines are only guides for the eyes. Optimizations have been performed for $T_{\lambda}=\lambda T_{F}$ with $\lambda \in\{1 / 80,1 / 40,1 / 20,1 / 10,1 / 5,1 / 2,1\}$.

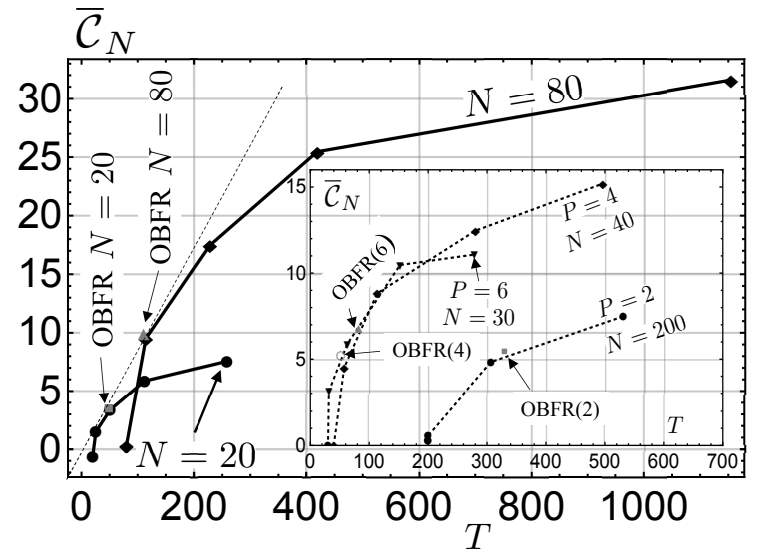

Fig. 4. Optimized criterion $\overline{\mathcal{C}}_{N}$ (Eq. (12)) as a function of the mean measuring time $T$ for classification between 4 classes with spectra with exponent $\alpha=1.0$ with $P=4 \mathrm{OBF}$ and for two $N$ values. The results obtained with maximization of $\overline{\mathcal{C}}_{N} / T$ are also reported (OBFR). Optimizations have been performed for $T_{\lambda}=\lambda T_{F}$ with $\lambda \in\{1 / 20,1 / 10,1 / 5,1 / 2,1\}$.

Insert: Idem but for several $N$ and $P$ (number of $\mathrm{OBF}$ ) values.

The method analyzed in this Letter can be adapted to a number $M$ of species larger that 2 . When each of the $M$ species has the same probability $1 / M$ to occur the classification error probability $\mathcal{P}(\mathcal{E})$ satisfies (see the Appendix):

$$
\mathcal{P}(\mathcal{E}) \leq \frac{1}{2} e^{-\overline{\mathcal{C}}_{N}}
$$

with:

$$
\overline{\mathcal{C}}_{N}=-\log \left[\frac{2}{M} \sum_{v} \sum_{u>v}\left(\boldsymbol{q}_{u} \cdot \boldsymbol{q}_{v}\right)^{N}\right]
$$

It can be noticed that, when $M=2, \overline{\mathcal{C}}_{N}=\mathcal{B}_{u, v}$ but when $M>$ $2, \overline{\mathcal{C}}_{N}$ is not a mean value of Bhattacharyya distances and can possibly be negative.

Analogous results to the ones obtained for $M=2$ in Fig.2 are reported in the insert of this Figure for $M=P=4$ (i.e. for four classes and simulation of four OBF) and for $N=50$. In that case, $\left(n_{1}, n_{2}, n_{3}, n_{4}\right)$ has a multinomial distribution with parameters $p_{1}^{(u)}, p_{2}^{(u)}, p_{3}^{(u)}, p_{4}^{(u)}$ for species $u$. The classification error probabilities have been estimated with $10^{3}$ realizations of random variables distributed with multinomial distributions and when each class has the same probability to occur. For these numerical simulations, $p_{m}^{u}=\frac{y_{m}^{u}}{\sum_{m=1}^{p} y_{m}^{u}}$, where $y_{m}^{u}$ are realizations of random numbers uniformly distributed in $[0,1]$ and $N_{p}=50$. The generalized Bhattacharyya bound $\frac{1}{M} \sum_{v} \sum_{u>v}\left(\boldsymbol{q}_{u} \cdot \boldsymbol{q}_{v}\right)^{N}$ is also reported in the insert of Fig.2, showing a similar behavior to the one with two classes.

Analogous results to the ones of Fig. 3 are reported in Fig.4 when $\alpha=1$ and for two different values of the imposed number of photons $(N=20$ and $N=80)$. In that case, the optimized criterion is $\overline{\mathcal{C}}_{N}$ with several maximal imposed value $T_{\lambda}$ of $T$. The optimization process is analogous to the one with $P=2$. The $\mathrm{OBF}$ are initialized with binary random values but with the constraint that $\sum_{m=1}^{P} F_{m}\left(v_{k}\right)=1$. Then, for each $v_{k}$, a new value of $\left[F_{1}\left(v_{k}\right), . ., F_{P}\left(v_{k}\right)\right]$ is chosen equal to $\left(a_{1}, a_{2}, . . a_{P}\right)$ where $a_{i}$ is a random value equal to 0 or 1 and so that $\sum_{i=1}^{P} a_{i} \leq 1$ (i.e. only one value $F_{m}\left(v_{k}\right)$ can be equal to 1$)$. This new value is accepted 
if it increases $\overline{\mathcal{C}}_{N}$ and satisfies $T=\frac{1}{M} \sum_{m=1}^{M} \tau_{m} \leq T_{\lambda}$. The number of iterations of the optimization process was set equal to 4000. Fig.4 illustrates the ability of the discussed method to be applied with several classes. It has nevertheless to be noticed that $\overline{\mathcal{C}}_{N} / T$ is no more independent of $N$. Thus maximizing $\overline{\mathcal{C}}_{N} / T$ for two different values of $N$ could in principle lead to OBF that correspond to different trade-off (denoted OBFR in Fig.4). This is indeed what can be observed since the optimization for respectively $N=20$ and $N=80$ leads respectively, for these examples, to $\overline{\mathcal{C}}_{N} / T=0.0701$ and $\overline{\mathcal{C}}_{N} / T=0.0844$.

It is not necessary to impose $P=M$ (i.e. equal number of OBF and of classes). Results with different values of $P(P \in$ $\{2,4,6\}$ ) when $M=4$ are reported in the insert of Fig. 4 for $\alpha=1$. For these examples the number of iterations has been chosen equal to $10^{3} \mathrm{P}$ so that each value $F_{m}\left(v_{k}\right)$ for different $k$ and $m$ values has been tested in average the same number of times. It can be seen that $P=2$ is not very efficient. Indeed, for approximately the same $\overline{\mathcal{C}}_{N}$ it leads to a large value of $T$. In other words, it leads to a mean number of equivalent emitted photons before the OBF, larger than the one obtained with $P=4$ or 6. It has nevertheless to be noticed that classification with $M$ species and $P<M$ are possible with low error probability at the expense of larger mean equivalent number of emitted photons before the OBF than when $P=M$.

In conclusion, the discussed method of this Letter presents several properties that can be very useful for practical applications. It leads to probabilities of classification error that can be upper bounded without knowing a priori the mean number of emitted photons but only knowing the shape of the normalized Raman spectra. It thus allows one to easily determine the total number of measured photons to guaranty that the classification error probability is smaller than an apriori fixed value. Mathematically orthogonal binary filters can be designed using a simple criterion obtained with the Bhattacharyya bound.

There exist many perspectives to this work. It will be interesting to develop more efficient OBF design methods since the obtained results are sensitive to the initialization of the optimization algorithm. However, this is not the central problem for the study of this Letter since the proposed approach can be implemented with any OBF. Thus, the most promising perspective is probably to implement optically the described approach of this Letter. The assumption that the energy of each frequency band can be sent to a specified detector although difficult is interesting in order not to loose too much efficiency in comparison to standard Raman measurements. It has nevertheless to be taken into account that simpler optical implementations may be considered for some applications since only few photons are necessary to get very low classification error probabilities with spectra that are not too much similar. It will be also interesting to analyze if such a technique can be used for other applications for classification based on other measurements than Raman spectrum analysis.

\section{APPENDIX}

Let $\mathcal{P}_{u \mid v}^{(M)}$ denote the classification error probability to decide that the observed spectra is due to species $u$ while it is due to species $v$. Let $t_{u}(\boldsymbol{n})=\sum_{m=1}^{P} n_{m} \log \left(p_{m}^{(u)}\right)$ and $\mathcal{R}_{u}^{(M)}=$ $\left\{\boldsymbol{n} \mid \sum_{m} n_{m}=N\right.$ and $\left.t_{u}(\boldsymbol{n}) \geq t_{w}(\boldsymbol{n}), \forall w \neq u\right\}$. Then $\mathcal{P}_{u \mid v}^{(M)}=$ $\sum_{\boldsymbol{n} \in \mathcal{R}_{u}^{(M)}} P(\boldsymbol{n} \mid v, N)$. Clearly, $\mathcal{P}_{u \mid v}^{(M)} \leq \mathcal{P}_{u \mid v}^{(2)}$ where $\mathcal{P}_{u \mid v}^{(2)}=$ $\sum_{\boldsymbol{n} \in \mathcal{R}_{u \mid v}^{(2)}} P(\boldsymbol{n} \mid v, N)$ with $\mathcal{R}_{u \mid v}^{(2)}=\left\{\boldsymbol{n} \mid \sum_{m} n_{m}=N\right.$ and $t_{u}(\boldsymbol{n}) \geq$ $\left.t_{v}(\boldsymbol{n})\right\}$ that corresponds to a two classes problem. But, the error probability $\mathcal{P}_{u, v}(\mathcal{E})=\frac{1}{2}\left[\mathcal{P}_{u \mid v}^{(2)}+\mathcal{P}_{v \mid u}^{(2)}\right]$ is upper bounded by $\frac{1}{2} e^{-\mathcal{B}_{u, v}}$. With $M$ equally probable species, (i.e. so that the apriori probability to observe species $v$ is $P(v)=1 / M$ ) the mean error probability is $\mathcal{P}(\mathcal{E})=\sum_{u, v \mid u \neq v} \mathcal{P}_{u \mid v}^{(M)} P(v)$ and thus satisfies:

$$
\mathcal{P}(\mathcal{E}) \leq \frac{1}{M} \sum_{u, v \mid u \neq v} \mathcal{P}_{u \mid v}^{(2)}=\frac{1}{2 M} \sum_{u, v \mid u \neq v}\left[\mathcal{P}_{u \mid v}^{(2)}+\mathcal{P}_{v \mid u}^{(2)}\right]
$$

that leads to Eq. (11) and Eq. (12).

\section{ACKNOWLEDGMENTS}

The authors thanks Camille Scotté and Hervé Rigneault for fruitful discussions.

\section{REFERENCES}

1. R. A. DeVerse, R. M. Hammaker and W. G. Fateley, Appl. Spectrosc. 54, 1751, (2000).

2. N.T. Quyen, M. D. Jouan, N. Q. Dao, E. Da Silva and D. A. Phuong, Appl. Spectrosc. 62, 273 (2008).

3. B. M. Davis, A. J. Hemphill, D. Cebeci Maltas, M. A. Zipper, P. Wang and D. Ben-Amotz, Anal. Chem. 83, 5086, (2011).

4. D. S. Wilcox, G. T. Buzzard, B. J. Lucier, P. Wang and D. Ben-Amotz, Anal. Chim. Acta 755, 17 (2012).

5. G. T. Buzzard and B. J. Lucier, Proc. SPIE 8657, 865707 (2013).

6. D. S. Wilcox, G. T. Buzzard, B. J. Lucier, O. G. Rehrauer, P. Wang and D. Ben-Amotz, Analyst, 138 4982, (2013).

7. P. Berto, C. Scotté, F. Galland, H. Rigneault and H. B. de Aguiar. Opt. Lett. 9, 1696, (2017).

8. C. Scotté, H. B. de Aguiar, D. Marguet, E. M. Green, P. Bouzy, S. Vergnole, C. P. Winlove, N. Stone, and H. Rigneault, Anal. Chem. 12 7197, (2018).

9. O. G. Rehrauer, V. C. Dinh, B. R. Mankani, G. T. Buzzard, B. J. Lucier and D. Ben-Amotz, Appl. Spectrosc. 72, 69 (2018).

10. C. Scotté, S. Sivankutty, P. Stockton, R. A. Bartels, and H. Rigneault, Optics Letters 44, 1936 (2019).

11. D. Cebeci, B. Mankani, and D. Ben-Amotz, Journal of Imaging 5, 1 (2019).

12. Ph. Réfrégier, C. Scotté, H. B. de Aguiar, H. Rigneault and F. Galland, J. Opt. Soc. Am. A 35, 125 (2018).

13. P. Refregier and F. Galland. Opt. Letters, 44, 2228 (2019)

14. C. W. Therrien, "Decision Estimation and Classification: An Introduction to Pattern Recognition and Related Topics," (Wiley, New York, 1989), pp. 29, and pp.144-146.

15. F. Goudail, N. Roux, and Ph. Refregier, Opt.Lett. 28, 81 (2003).

16. F. Goudail, Ph. Refregier and G. Delyon, J. Opt. Soc. Am. A 21, 1231, (2004). 


\section{REFERENCES}

1. DeVerse, R. A., Hammaker, R. M. and Fateley, W. G., "Realization of the Hadamard Multiplex Advantage Using a Programmable Optical Mask in a Dispersive Flat-Field Near-Infrared Spectrometer," Appl. Spectrosc., 54, 1751-1758, (2000).

2. Quyen, N.T., Jouan, M. D., Dao, N. Q., Da Silva, E. and Phuong D. A. "New Raman spectrometer using a digital micromirror device and a photomultiplier tube detector for rapid on-line industrial analysis. Part II: Choice of analytical methods," Appl. Spectroscop., 62, 273-278 (2008).

3. Davis, B. M., Hemphill, A. J., Cebeci Maltas, D., Zipper, M. A.; Wang, P. and Ben-Amotz, D., "Multivariate hyperspectral Raman imaging using compressive detection," Anal. Chem., 83, 5086-5092, (2011).

4. Wilcox, D. S.; Buzzard, G. T.; Lucier, B. J.; Wang, P. and Ben-Amotz, D., "Photon level chemical classification using digital compressive detection," Anal. Chim. Acta, 755, 17-27 (2012).

5. Buzzard, G. T. and Lucier, B. J., Optimal filters for high-speed compressive detection in spectroscopy," Proc. SPIE 8657, 865707 (2013).

6. Wilcox, D. S.; Buzzard, G. T.; Lucier, B. J.; Rehrauer, O. G.; Wang, P. and Ben-Amotz, D., "Digital compressive chemical quantitation and hyperspectral imaging," Analyst, 138, 4982-4990, (2013).

7. P. Berto, C. Scotté, F. Galland, H. Rigneault and H. B. de Aguiar. "Programmable single-pixel-based broadband stimulated Raman scattering," Opt. Lett., 9, 1696-1699, (2017).

8. C. Scotté, H. B. de Aguiar, D. Marguet, E. M. Green, P. Bouzy, S. Vergnole, C. P. Winlove, N. Stone, and H. Rigneault, "Assessment of compressive Raman versus hyperspectral Raman for microcalcification chemical imaging," Anal. Chem. (2018).

9. O. G. Rehrauer, V. C. Dinh, B. R. Mankani, G. T. Buzzard, B. J. Lucier, and D. Ben-Amotz, "Binary complementary filters for compressive Raman spectroscopy," Appl. Spectrosc. 72, 69-78 (2018).

10. C. Scotté, S. Sivankutty, P. Stockton, R. A. Bartels, and H. Rigneault, "Compressive raman imaging with spatial frequency modulated illumination," Optics Letters 44, 1936-1939 (2019).

11. D. Cebeci, B. Mankani, and D. Ben-Amotz, "Recent trends in compressive raman spectroscopy using dmd-based binary detection," Journal of Imaging 5, 1 (2019).

12. Réfrégier, Ph., Scotté, C., de Aguiar, H. B., Rigneault, H. and Galland, F. "Precision of proportion estimation with binary compressed Raman spectrum," J. Opt. Soc. Am. A 35, 125-134 (2018).

13. P. Refregier and F. Galland. 'Bhattacharyya bound for Raman spectrum classification with a couple of binary filters," Opt. Letters, 44, 2228-2231 (2019)

14. C. W. Therrien, "Decision Estimation and Classification: An Introduction to Pattern Recognition and Related Topics," (Wiley, New York, 1989), pp. 29-35, and pp.144-146.

15. F. Goudail, N. Roux, and Ph. Refregier, "Performance parameters for detection in low-flux coherent images," Opt.Lett. 28, 81-83 (2003).

16. F. Goudail, P. Refregier, and G. Delyon, "Bhattacharyya measure as a contrast parameter for statistical processing of noisy optical images," J. Opt. Soc. Am. A 21, 1231-1240, (2004). 